\title{
Inherent Work Suit Buoyancy Distribution: Effects on Lifejacket Self-Righting Performance
}

Martin J. Barwood, Geoffrey M. Long, Heather Lunt, and Michael J. Tipton

Introduction: Accidental immersion in cold water is an occupational risk. Work suits and life jackets (LU) should work effectively in combination to keep the airway clear of the water (freeboard) and enable selfrighting. We hypothesized that inherent buoyancy, in the suit or $L$, would be beneficial for enabling freeboard, but its distribution may influence LJ self-righting.

Methods: Six participants consented to complete nine immersions. Suits and $L J$ tested were: flotation suit (FLOAT; $85 \mathrm{~N}$ inherent buoyancy); oilskins 1 (OS-1) and 2 (OS-2), both with no inherent buoyancy; LJs (inherent buoyancy/buoyancy after inflation/total buoyancy), LJ-1 50/150/200 N, LJ-2 0/290/290 N, LJ-3 $80 / 190 / 270 \mathrm{~N}$. Once dressed, the subject entered an immersion pool where uninflated freeboard, selfrighting performance, and inflated freeboard were measured. Data were compared using Friedman's test to the 0.05 alpha level.

Results: All suits and LJs enabled uninflated and inflated freeboard, but differences were seen between the suits and LJs. Self-righting was achieved on 43 of 54 occasions, irrespective of suit or L. On all occasions that self-righting was not achieved, this occurred in an $L$ that included inherent buoyancy $(11 / 54$ occasions). Of these 11 failures, 8 occurred (73\% of occasions) when the FLOAT suit was being worn.

Discussion: LJs that included inherent buoyancy, that are certified as effective on their own, worked less effectively from the perspective of selfrighting in combination with a work suit that also included inherent buoyancy. Equipment that is approved for use in the workplace should be tested in combination to ensure adequate performance in an emergency scenario.

Accidental cold-water immersion (CWI) is a risk factor for aviators and for persons who work on or around cold water. On immersion, a cascade of life-threatening physiological responses is evoked that are collectively described as the "cold shock response" [CSR ( 7 )]. The CSR is driven by a rapid change in skin temperature and culminates in significant cardiovascular strain and a loss of respiratory control that pose a threat to otherwise healthy individuals ( 7 ). These responses make swimming a short distance to safety or executing a life-saving strategy problematic ( 4 ). The CSR diminishes after the first few minutes of 
immersion ( 7 ), following which a survival plan could be executed. Accordingly, it is prudent to defend the airway if accidental immersion should occur.

It may be possible to minimize skin temperature reductions on water entry by wearing a protective immersion suit ( 9 ) and to defend the airway by means of inherent buoyancy to the suit or by donning a life jacket [ $L(1)]$. Any innovation that increases the distance between the water level and opening of the mouth (i.e., freeboard) will reduce the risk of water entering the airway ( 1 ). The ergonomics of such devices should be balanced against the requirement to enable occupational performance while meeting the demands of the user should accidental immersion occur. Consequently, an evidence-based approach should be sought to justify the use of protective clothing ensembles.

There are few published studies that have reported whether clothing that is designed to work independently to agreed standards works similarly well when combined with other life-saving appliances (LSA), as may be the scenario in the occupational setting. Indeed, it is not an ISO requirement for LJs to be tested in combination with other LSA ( 6 ). Armstrong et al. ( 1 ) tested three aviation and five marine LJs in combination with three immersion suits. The extent of the $\mathrm{L}$ buoyancy ranged from $158 \mathrm{~N}$ to $315 \mathrm{~N}$ and comprised single-lobe, twin-lobe, and asymmetric lobe designs. The immersion suits ranged from no deliberate inherent buoyancy through to a CO 2 -inflated helicopter passenger suit, although no explicit buoyancy by suit was reported. They tested these combinations in calm conditions in a laboratory and in representative rough conditions in open water. They concluded that single-lobe $\amalg$ that included high inherent buoyancy provide good self-righting performance. In contrast, their data suggested that twin-lobe LJ, when worn over an immersion suit, offered poor selfrighting performance. However, these data were collected using a manikin and in immersion suits and Ls that were intended for offshore deployment.

One predominantly inshore occupation that tends toward using a $L J$ in combination with a protective work suit is the UK and international sea farming industry. Work suits differ from 'immersion suits' in that they are not fully sealed against water ingress, which increases the chance of a maximal CSR, but this is balanced against the need for occupational performance and to minimize thermal burden (i.e., avoiding a sealed barrier to heat loss). Moreover, any buoyancy provided by trapped air is likely to be minimized in a work suit compared to an immersion suit per se. Traditionally the sea farming industry has used an oilskin protective suit which contains no inherent buoyancy. However, work suits that include inherent buoyancy, independent of $\mathrm{L}$, have become available for use in such circumstances. These suits negate the immediate need for a $L J$ that also includes inherent buoyancy and may enable freeboard in the conscious casualty. However, if the buoyancy in each ensemble is not distributed in a complementary and ergonomic way, it may provide resistance to turning moment if the casualty is immersed face down and the $L$ is required to self-right the victim ( 1 ). 
Accordingly, this study sought to investigate the efficacy of work suit and $L$ combinations for freeboard performance and self-righting capability on immersion. It was hypothesized that a work suit that included inherent buoyancy would enable greater freeboard than those suits that included no inherent buoyancy $(\mathrm{H}$ 1). However, it was also hypothesized that the suit that included inherent buoyancy may alter the selfrighting characteristics when combined with an $\mathrm{L}$ that included inherent buoyancy $(\mathrm{H} 2)$.

\section{METHODS}

Subjects

The study was approved by the Biosciences Research Ethics Committee and the subjects gave written informed consent. Six nonsmoking subjects (five men, one women) volunteered for the experiment [mean (SD); age 20 (2) yr; height 1.77 (0.11) m; mass 80.02 (11.64) kg]. The subjects were selected to be representative of the physiological characteristics of the working population of the sea farming community (supplied by the funding organization) and were within $10 \%$ of a 'normal weight' body mass index (BMI) $[25.8(3.6)]$.

\section{Experimental Design}

The study used a within-subject repeated measures design. The subjects visited the laboratory on one occasion and completed a total of nine immersions in a randomized order in each of the three work suits in combination with each LJ. During these immersions initial buoyancy was first examined prior to jacket inflation (uninflated freeboard), followed by a self-righting performance test, and concluding with a further freeboard measurement (inflated freeboard). All tests were conducted in thermoneutral water $\left(35^{\circ} \mathrm{C}\right)$.

\section{Procedure}

Following arrival at the laboratory, each subject changed into a swimming costume. They completed a measurement of height $(\mathrm{m})$ and mass $(\mathrm{kg})$ using a stadiometer (Bodycare Stadiometer, Leicester, UK) and calibrated weighing scales (OHAUS digital weighing scales, Parsippany, NJ). The subject then donned a set of standard clothing comprising a woolen pullover, long sleeved cotton shirt, cotton trousers, woolen socks, and Wellington boots.

Each subject was then familiarized with work suit donning and $L J$ deployment prior to any testing in water. Once dressed, they entered an environmental chamber with a 1.5-m deep immersion pool, mounted the steps to the pool and lowered themselves into the water in order to prewet the under clothing to standardize the saturation of the clothing under each suit. They then left the water and put on a work suit and $L$. They then reentered the water and purged the suit of air by performing a 30-s forced immersion to 
the suit neck seal. The subject was instructed to roll on to their back, with the jacket uninflated, in order that $\mathrm{L}$ uninflated freeboard could be measured. Freeboard measurement was performed using a retractable measuring cylinder positioned above the immersion pool that rested near the participant's lips cross referenced against a measuring rule ( 2 ).

The subject then completed the self-righting test on $L$ inflation. Following an initial practice attempt the participant fell forward from a standing position until their face entered the water. They breath-held and kept their arms by their sides, one hand holding the inflation toggle of the $L$ in preparation for inflation. They drifted for a few seconds and then, on the command 'Fire,' pulled sharply down in one single action to inflate the $L$. The performance of self-righting was measured by stopwatch as the time taken from initial inflation to the point at which the airway cleared the water. Subsequently the inflated freeboard was measured using the same technique as prior to inflation. Lastly, the participant returned to one end of the pool and completed the same drifting procedure to see if the $L$ enabled self-righting once inflated. The subject then exited the immersion pool and changed the ensemble for the next test. Once all tests were complete the participant rated each clothing combination on a 5-point Likert scale of 0 (poorest possible performance) to 5 (best possible performance). The participant then showered, changed, and left the laboratory.

\section{Equipment}

1) One-piece flotation suit (FLOAT) with an inherent $85 \mathrm{~N}$ of buoyancy distributed around the legs, arms, and torso in the form of PVC flotation material. EN393 approved, ISO 15,027-1 certified. Approximate weight: $2.2 \mathrm{~kg}$.

2) Two-piece, heavy-duty, cotton backed PVC oilskins (OS-1) with no inherent buoyancy. Approximate weight: $3.0 \mathrm{~kg}$.

3) Two-piece, heavy-duty, cotton backed PVC oilskins (OS-2) with no inherent buoyancy. Approximate weight: $3.1 \mathrm{~kg}$.

To avoid premature inflation, each $L$ auto inflation device was removed prior to jacket packing. Between tests, all LJs were repacked by trained personnel in accordance with manufacturer's instructions.

1) LJ-1: $50 \mathrm{~N}$ of uninflated inherent buoyancy distributed around the front and back of the jacket with the addition of a further 150-N buoyancy (twin lobe) on jacket inflation ( $50 \mathrm{~N}$ inherent/150 N on inflation/200 $\mathrm{N}$ total).

2) L-2: no uninflated inherent buoyancy, but with the addition of $290 \mathrm{~N}$ of buoyancy (twin lobe), on inflation (0/290/290). 
3) LJ-3: $80 \mathrm{~N}$ of inherent uninflated buoyancy distributed around the front and back of the jacket with the addition of $190 \mathrm{~N}$ (twin lobe) on jacket inflation (80/190/270).

\section{Statistical Analyses}

A preset criteria for self-righting performance standard was set of $5 \mathrm{~s}$ for the airway to clear the water ( 3 ). A time in excess of $5 \mathrm{~s}$ was considered a self-righting failure ( 3 ). Where failure to self-right was observed, a time of $5 \mathrm{~s}$ was allocated to the data set to enable statistical comparisons between conditions. Mean (SD) were calculated for self-right performance (absolute number and percentage) and duration, uninflated freeboard, inflated freeboard, and drifting self-right performance.

Data, grouped by suit and $\amalg$, were assessed for normality of distribution using a Kolmogorov-Smirnov test, but were not normally distributed. Therefore, data were compared using a nonparametric Friedman test grouped by work suit and $\amalg$ type. Where significant effects were evident, post hoc pairwise comparisons were made using the Wilcoxon signed rank test and Bonferroni adjustment for multiple comparisons. For all statistical tests a level was set at 0.05. Data are presented as mean (SD). All statistical tests were conducted using SPSS version 20 (Chicago, IL).

\section{RESULTS}

Irrespective of $\mathrm{L}$ and prior to its inflation, freeboard was achieved on all occasions and this averaged 10.2 (1.7) $\mathrm{cm}, 8.8(5.0) \mathrm{cm}$, and $8.9(3.3) \mathrm{cm}$ in the FLOAT, OS-1, and OS-2, respectively, which were not different $(P=0.158$; see Fig. 1). Once the jacket had been inflated, the freeboard height increased to $17.1(1.9) \mathrm{cm}$, $16.3(3.5) \mathrm{cm}$, and $17.1(2.6) \mathrm{cm}$, respectively, which were not different between suits $(P=0.834)$. When self-righting was attempted it took significantly longer $(P=0.007)$ when wearing the FLOAT suit [3.3 (1.7) s] compared to both OS-1 [2.4 (1.2) s; P = 0.013] and OS-2 [2.6 (1.5) s; P = 0.003]; OS-1 and OS-2 were not different ( $P=0.602$; see Fig. 2 ). As a consequence of the slower self-righting, failure to self-right was seen more frequently in the FLOAT suit (10/18 occasions; $56 \%$ success rate) in contrast to OS-1 (17/18 occasions; $94 \%$ success rate) and OS-2 (16/18 occasions; $89 \%$ success rate). The drifting selfrighting statistics were similar to those as on inflation (FLOAT: $11 / 18$ occasions, $61 \%$ success rate; OS-1: $18 / 18$ occasions, $100 \%$ success rate; OS-2: $17 / 18$ occasions, $94 \%$ success rate). 


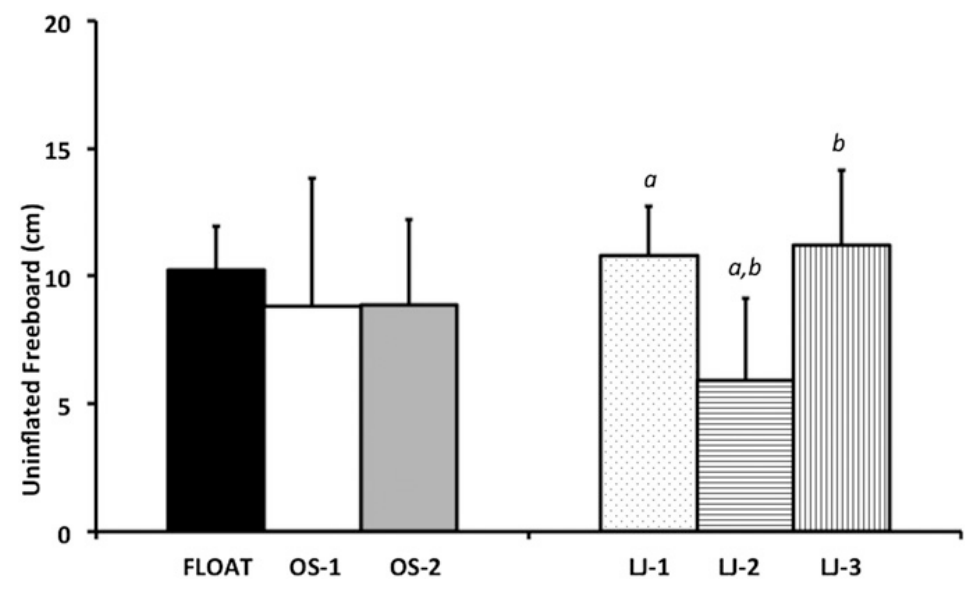

Fig. 1. Mean (SD) uninflated freeboard performance in each suit and each $\mathrm{J} ; \mathrm{a}$ and $\mathrm{b}$ indicate significant differences between marked conditions (within suit and $L)(N=6)$.

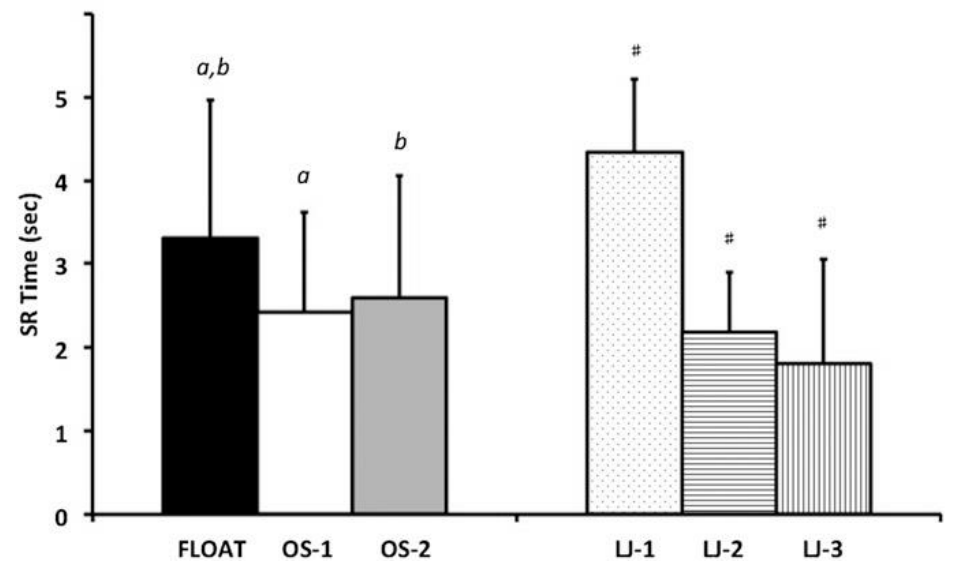

Fig. 2. Mean (SD) self-righting performance in each suit and each $\mathrm{L}$; $a$ and $b$ indicate significant differences between marked conditions (within suit and $\mathrm{LJ}$ ); $\circledast$ indicates significant differences between all conditions $(N=6)$.

Irrespective of work suit worn and prior to $L$ inflation, freeboard was achieved in all clothing combinations. There were significant differences $(P=0.001)$ in the extent to which this was seen with LJ-1 and LJ-3 (i.e., those which included inherent buoyancy), both having significantly greater associated freeboard [10.8 (1.9) $\mathrm{cm}, P=0.001$, and $11.2(2.9) \mathrm{cm}, P=0.001]$ than $\mathrm{LJ}-2$, which included no inherent buoyancy [5.9 (3.2) cm]; LJ-1 and LJ-3 were not different $(P=0.887)$. After jacket inflation, significant differences in inflated freeboard were also evident $(P=0.008)$, but not in the same direction as prior to inflation. LJ-3 [18.5 (2.5) $\mathrm{cm}$ ] showed significantly greater freeboard than both L-2 [16.7 (1.8) cm; $P=0.008]$ and LJ-1 [15.3 (2.8) cm; $P=0.004]$; LJ-1 and LJ-2 were not different $(P=0.109)$. From the perspective of self-righting time, all jackets were different $(P=0.001)$, with $L J-1$ evidently the slowest $[4.3(0.9) \mathrm{s}]$ compared to $\mathrm{LJ}-2[2.2(0.7) \mathrm{s} ; P=$ 0.001], which were both slower than LJ-3 [1.8 (1.2) s; $P=0.001$ and $P=0.029]$; see Fig. 1 . The consequence 
of the slower self-righting in LJ-1 was fewer successful self-righting performances (9/18 occasions; 50\% success rate). However, despite being quicker to achieve self-righting, LJ-3 achieved this on fewer occasions (16/18 occasions; $89 \%$ success rate) than LJ-2 (18/18 occasions; $100 \%$ success rate). After $L J$ inflation (i.e., in the drifting self-righting test) the performance statistics were similar for each jacket (LJ-1: 9/18 occasions, $50 \%$ success rate; LJ-2: 18/18 occasions, $100 \%$ success rate; LJ-3: 17/18 occasions, $94 \%$ success rate). Collectively, these data suggest that, on and following inflation, the prospect of achieving self-righting were similar.

On all occasions that self-righting was not achieved (11/54 occasions), this occurred in an $\amalg$ that included inherent buoyancy (i.e., LJ-1 or LJ-3). Of these 11 failures, 8 occurred when the FLOAT suit was being worn (73\% of failures). The remaining three failures were seen when LJ-1 was being worn and in the participants who tended toward being of greater height and mass (i.e., equal to or above $1.85 \mathrm{~m}$ and $80 \mathrm{~kg}$ ). When appraising their favored combination, four participants selected the FLOAT suit with LJ-2; the remaining two participants selected OS-1 and OS-2, also in combination with LJ-2 (i.e., the $L$ that included no inherent buoyancy).

\section{DISCUSSION}

This study investigated the efficacy of work suit and $L$ combinations for freeboard performance and selfrighting capability on immersion. Irrespective of clothing combination, our data suggest that LJs that include inherent buoyancy provide significantly greater freeboard than those that do not prior to $L J$ inflation, but that the $\amalg$ ergonomics are the primary driver of the success of self-righting and magnitude of freeboard thereafter. However, work suits that include inherent buoyancy do not necessarily culminate in greater freeboard than those that do not; $\mathrm{H} 1$ is, therefore, rejected. It is also evident from our data that $\mathrm{LJ}$ that include inherent buoyancy combined with a work suit that also includes some inherent buoyancy increase the risk of slower self-righting performance or failure to self-right. After $\mathrm{LJ}$ inflation the selfrighting characteristics were also similar; $\mathrm{H} 2$ can, therefore, be accepted.

Our data are in agreement with Armstrong et al. ( 1 ), who included test combinations that were similar to those reported here. Armstrong et al. ( 1 ) tested uninsulated and insulated immersion suits which may be comparable to an oilskin work suit and a FLOAT work suit, respectively (i.e., they exclude and include inherent buoyancy). They suggested that the uninsulated immersion suit does not necessarily impair selfrighting performance, whereas an immersion suit, particularly in combination with a twin-lobe $L$, offers poor performance. Our data concur in that a large proportion of successful self-righting performances were seen when the oilskin work suits were worn (77\% of successes), irrespective of $L$, whereas the predominance of self-righting failures were seen when the FLOAT suit was worn and in combination with an $\mathrm{LJ}$ that included inherent buoyancy. It is possible that differences in flotation angle are implicated in this 
difference in performance. When successful self-righting was seen with this combination, performance tended to be slower [3.8 (1.7) s] than when the FLOAT suit was worn with an $L$ that did not have any inherent buoyancy [2.6 (1.9) s] or in contrast to any combination of oilskin work suit with an LJ [OS-1: 2.4 (1.2) s; OS-2: 2.6 (1.5) s; see Fig. 2]. Collectively, these data suggest that a FLOAT work suit combined with an LJ with inherent buoyancy do not comprise complementary float characteristics when self-righting is the primary goal.

The importance of self-righting is controversial as it is argued that most victims will be conscious on water entry and will be able to right themselves ( 1 ). If this is the case and prolonged immersion ensues, it is the thermal insulation characteristics of the work suits that may ultimately be important in determining survival. We also tested the work suits over a $45-\min 10^{\circ} \mathrm{C} \mathrm{CWI}$ and found the insulation of the FLOAT suit to be superior to an oilskin suit (data not shown), which contrasts performance in the self-righting tests. These data support the idea that consideration of all aspects of the responses to CWI (initial, short, and long-term) should underpin recommendations for appropriate clothing combinations in the workplace. Self-righting performance becomes important when the immersed victim is unconscious or for those who are transiently capsized by a wave. When poor self-righting performance was seen, we speculate that the natural flotation angle may be influenced and that the positioning of the inherent buoyancy within the suit and $L J$ provides resistance to the turning moment that is required to self-right a casualty. It is also possible that the $L$ design and the victim's physical characteristics play a role in the success of self-righting. In the case of the former, Herrman ( 5 ) suggested that $L J$ with a large central chest lobe are likely to produce the greatest turning force by way of having a relatively longer lever arm to that of a double-lobed LJ. In the latter case we cite our evidence that the three failures to self-right when the oilskin work suit was worn were restricted to subjects who were relatively taller and heavier.

Clearly, our study is not without limitation. The tests were conducted in calm, warm water in a laboratory. A real-life emergency situation could include turbulent water conditions (i.e., waves) and increased wind speed. Our set of test conditions represents a 'best case scenario,' but it is possible that momentary turbulent water and wind could either aid or impair self-righting. Armstrong et al. ( 1 ), who did test in turbulent conditions (i.e., wave heights 0.5 to $2.6 \mathrm{~m}$; wind speeds up to $17 \mathrm{~m} \cdot \mathrm{s} 21$ ), suggested that turbulent weather and sea conditions impaired immersion suit and $L J$ performance in similar tests. Armstrong et al. ( 1 ) also showed that at wave heights above $0.5 \mathrm{~m}$, there was an increase in the frequency of periodic wave splash to the face. Lastly, it is also possible that cold water, by inducing the CSR, could induce involuntary body movements that could also alter self-righting performance.

In summary, our data suggest that work suits that include inherent buoyancy when combined with LJs that also include inherent buoyancy increase the risk of self-righting failure on immersion. Clearly, LSA that are 
shown to work independently do not necessarily work as effectively in combination and test standards should consider the interaction of such devices ( 8 ). Work wear combinations should be tested in advance of an emergency scenario to establish an evidence base for wear in occupations that include a daily risk of CWI if accidental death by drowning is to be avoided.

\section{ACKNOWLEDGMENTS}

The authors wish to acknowledge the contribution of Mr. Ian Wilson and Mr. Nigel Parkes, the subjects for their reliability and forbearance, and the technical support staff who worked on this project.

\section{REFERENCES}

1. Armstrong IJ, Bennett-Smith SC, Coleshaw SRK. Performance of immersion suit and lifejacket combinations at sea. Aberdeen, Scotland: Health and Safety Executive Offshore technology report; 1994. Report No: OTH 94428.

2. Barwood MJ, Bates V, Long G, Tipton MJ. "Float First: "trapped air between clothing layers significantly improves buoyancy after immersion. IJARE 2011; 5:147 - 63 .

3. European Committee for Standardisation (CEN). Lifejackets and personal buoyancy aids - lifejackets 275 N. Brussels, Belgium: European Standards Report; 1994. Report No: EN 399.

4. Golden FStC, Hardcastle PT, Pollard CE, Tipton MJ. Hyperventilation and swim failure in man in cold water. J Physiol 1986; 378: 94P.

5. Herrman R. Life-jackets: survival in heavy seas. Travel Medicine International 1991; 9: 77 - 80.

6. ISO Personal flotation devices - part 2: lifejackets performance level. 275 - Safety requirements. Geneva, Switzerland: International Standard Report; 2010. Report No: ISO 12402-2.

7. Tipton MJ. The initial responses to cold-water immersion in man. Clin Sci 1989; 77: 581 - 8.

8. Tipton MJ. The concept of an 'Integrated Survival System 'for protection against the responses associated with immersion in cold water. J R Nav Med Serv 1993; 79: 11 - 4. 
9. Tipton MJ, Golden FStC. The influence of regional insulation on the initial responses to cold immersion. Aviat Space Environ Med 1987; 58: 1192 - 6 . 